\title{
Effects of iron supplementation on dominant bacterial groups in the gut, faecal SCFA and gut inflammation: a randomised, placebo-controlled intervention trial in South African children
}

\author{
Alexandra Dostal ${ }^{1} \dagger$, Jeannine Baumgartner ${ }^{2,3} \dagger$, Nathalie Riesen ${ }^{1}$, Christophe Chassard ${ }^{1}$, \\ Cornelius M. Smuts ${ }^{3}$, Michael B. Zimmermann ${ }^{2}$ and Christophe Lacroix ${ }^{1 *}$ \\ ${ }^{1}$ Laboratory of Food Biotechnology, Institute of Food, Nutrition and Health, ETH Zurich, Schmelzbergstrasse 7, 8092 Zurich, \\ Switzerland \\ ${ }^{2}$ Laboratory of Human Nutrition, Institute of Food, Nutrition and Health, ETH Zurich, Schmelzbergstrasse 7, 8092 Zurich, \\ Switzerland \\ ${ }^{3}$ Centre of Excellence for Nutrition, North-West University, Potchefstroom Campus, South Africa \\ (Submitted 5 December 2013 - Final revision received 26 March 2014 - Accepted 14 April 2014 - First published online 11 June 2014)
}

\section{Abstract}

Fe supplementation is a common strategy to correct Fe-deficiency anaemia in children; however, it may modify the gut microbiota and increase the risk for enteropathogenic infection. In the present study, we studied the impact of Fe supplementation on the abundance of dominant bacterial groups in the gut, faecal SCFA concentration and gut inflammation in children living in rural South Africa. In a randomised, placebo-controlled intervention trial of 38 weeks, 6- to 11-year-old children with Fe deficiency received orally either tablets containing $50 \mathrm{mg} \mathrm{Fe}$ as $\mathrm{FeSO}_{4}$ ( $n$ 22) for $4 \mathrm{~d}$ /week or identical placebo ( $n$ 27). In addition, Fe-sufficient children ( $n$ 24) were included as a non-treated reference group. Faecal samples were analysed at baseline and at 2, 12 and 38 weeks to determine the effects of Fe supplementation on ten bacterial groups in the gut (quantitative PCR), faecal SCFA concentration (HPLC) and gut inflammation (faecal calprotectin concentration). At baseline, concentrations of bacterial groups in the gut, faecal SCFA and faecal calprotectin did not differ between Fe-deficient and Fe-sufficient children. Fe supplementation significantly improved Fe status in Fe-deficient children and did not significantly increase faecal calprotectin concentration. Moreover, no significant effect of Fe treatment or timextreatment interaction on the concentrations of bacterial groups in the gut or faecal SCFA was observed compared with the placebo treatment. Also, there were no significant differences observed in the concentrations of any of the bacterial target groups or faecal SCFA at 2,12 or 38 weeks between the three groups of children when correcting for baseline values. The present study suggests that in African children with a low enteropathogen burden, Fe status and dietary Fe supplementation did not significantly affect the dominant bacterial groups in the gut, faecal SCFA concentration or gut inflammation.

\section{Key words: Iron supplementation: Iron deficiency: Gut microbiota: Children}

Fe deficiency affects more than 2 billion people worldwide, and children, because they require high amounts of $\mathrm{Fe}$ for growth and development, are among the most vulnerable ${ }^{(1)}$. Fe deficiency can lead to Fe-deficiency anaemia and impair school performance and cognitive development in children $^{(2,3)}$. Thus, adequate dietary $\mathrm{Fe}$ availability for school-aged children is critical. In the past, the WHO recommended that children living in the regions with a high prevalence of $\mathrm{Fe}$ deficiency receive oral supplementation of $\mathrm{Fe}$ as $\mathrm{FeSO}_{4}^{(1,4,5)}$. This recommendation has been modified in recent years because of concerns that Fe supplementation may increase the risk for hospitalisations and mortality from infections $^{(6-9)}$
Supplemental Fe is poorly absorbed in the human gastrointestinal tract and most of the dose passes into the colon where it becomes available for the gut microbiota. The symbiotic bacteria in the gut provide the host with many beneficial functions, such as colonisation resistance from pathogens, immunomodulatory properties and degradation of indigestible compounds, while producing bacterial metabolites, such as SCFA, influencing host health and providing additional energy to host cells ${ }^{(10,11)}$. There are alterations in the composition of the gut microbiota in malnourished children in developing countries, and it has been proposed that the microbiota may contribute to the effects of nutritional deficiencies in these settings ${ }^{(12-16)}$. Several animal studies

Abbreviations: CRP, C-reactive protein; qPCR, quantitative PCR; SF, serum ferritin; TfR, serum transferrin receptor; ZnPP, zinc protoporphyrin.

*Corresponding author: C. Lacroix, fax + 414463214 03, email christophe.lacroix@hest.ethz.ch

† Both authors contributed equally to this work. 
have suggested that host Fe status and dietary Fe availability can influence the microbial ecosystem of the gut ${ }^{(17-22)}$, and that most bacteria in the gut have a requirement for $\mathrm{Fe}^{(23)}$.

However, the impact of Fe status and dietary Fe availability on the gut microbiota in humans is uncertain. A recent study in India has reported decreased numbers of lactobacilli in women with Fe-deficiency anaemia ${ }^{(24)}$. Furthermore, two studies in infants ${ }^{(25,26)}$ and one randomised placebocontrolled trial in Ivorian school children living in a rural area with a high prevalence of environmental pathogens ${ }^{(9)}$ have reported changes in the composition of the gut microbiota after Fe fortification. It has also been shown that the growth and infectivity of several enteropathogens can be promoted by Fe supplementation in vitro ${ }^{(27)}$ and also in hosts with Fe overload ${ }^{(28)}$. Both a dysbiosis of the gut microbiota, and hence a reduced barrier effect and colonisation resistance against pathogens ${ }^{(29)}$, and enhanced pathogen growth due to high-dose Fe supplementation could increase the risk for the development of diarrhoea. Indeed, a systematic review and a recent study in young children in Pakistan have found that Fe supplementation may increase the incidence of diarrhoea in children, especially in areas with a high prevalence of enteric pathogens ${ }^{(6,30)}$. Moreover, an increase in infection with other pathogens due to Fe supplementation could have an impact on the inflammatory preset of the host, which in turn might alter the composition of the gut microbiota and facilitate colonisation with enteropathogenic bacteria ${ }^{(8,29,31)}$

Based on these previous studies and observations, our hypothesis was that high-dose Fe supplementation and also host Fe status can affect the dominant commensal bacterial groups in the gut, their main metabolites and gut inflammation. This may lead to a potential dysbiosis of the gut microbiota with less protection against the establishment of environmental bacteria, such as enteropathogens, and a change in the degradation of dietary compounds. To test this hypothesis, we investigated the impact of oral supplementation of $\mathrm{Fe}$ as $\mathrm{FeSO}_{4}$ over a time period of 38 weeks on the concentrations of dominant bacterial groups in the gut, faecal SCFA and faecal calprotectin, a gut inflammation marker, in Fe-deficient school children living in rural South Africa compared with a placebo treatment without $\mathrm{FeSO}_{4}$ over the same time period. We also included non-treated Fe-sufficient children to investigate whether the abundance of dominant bacterial groups, faecal SCFA concentration and faecal calprotectin concentration differs between Fe-sufficient and Fe-deficient children.

\section{Subjects and methods}

\section{Study design}

Participants included in the present study were 6- to 11year-old children $(n$ 73) from two primary schools that serve low-income rural villages in the Province of KwaZulu-Natal in eastern South Africa (Fig. 1). A total of two groups of children were randomly selected from two arms of a $2 \times 2$ study design assessing the effects of Fe and $n-3$ fatty acid supplementation, alone and in combination, on cognition in school children, as reported previously ${ }^{(3)}$. One group received Fe supplements (Fe group, $n$ 22) and another group received placebo (placebo group, $n$ 27). As described previously, these children lived in a malaria-free region and fulfilled the following inclusion criteria: (1) 6 to 11 years of age; (2) $\mathrm{Hb}$ concentration $>80 \mathrm{~g} / \mathrm{l}$; (3) Fe deficient (serum ferritin (SF) concentration $<20 \mu \mathrm{g} / \mathrm{l}$ or zinc protoporphyrin (ZnPP) concentration $>70 \mu \mathrm{mol} / \mathrm{mol}$ haem or serum transferrin receptor (TfR) concentration $>8.3 \mathrm{mg} / \mathrm{l}$ ); (4) no chronic disease; (5) not using Fe supplements ${ }^{(3)}$.

Children in the Fe group were given orally one tablet containing $50 \mathrm{mg} \mathrm{Fe}$ as $\mathrm{FeSO}_{4}$ (Lomapharm; Paul Lohmann $\mathrm{GmbH})$ together with a fruit-flavoured and vitamin C-enriched (approximately $10 \mathrm{mg} /$ serving) beverage (200 $\mathrm{ml}$ ) for $4 \mathrm{~d} /$ week,

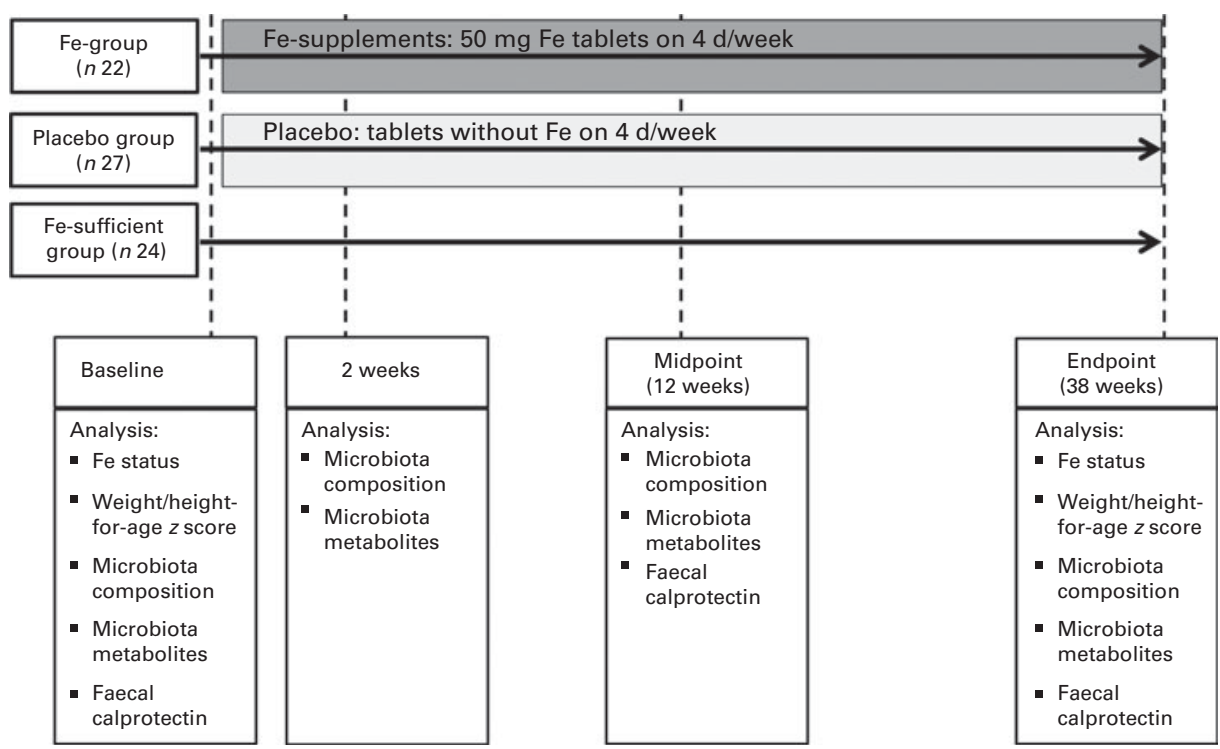

Fig. 1. Summary of the study design with interventions in the placebo and iron groups as well as the different sampling time points and the corresponding analysis. 
while those in the placebo group received an identical tablet without $\mathrm{Fe}$ and the beverage ${ }^{(3)}$. Trained fieldworkers (one fieldworker per group and school) directly supervised the tablet consumption and recorded compliance and selfreported illness symptoms while absent and present. Children in the placebo and Fe groups were dewormed with an oral dose of $400 \mathrm{mg}$ mebendazole (Be-Tabs Pharmaceuticals (Pyt) Limited) 3 and 15 weeks after the start of the intervention trial.

A third group of children (Fe-sufficient group, $n$ 24) with highest SF concentrations (without inflammation, C-reactive protein (CRP) concentration $<5 \mathrm{mg} / \mathrm{l}$ ) and thereafter lowest TfR and ZnPP concentrations was randomly selected out of 100 children from the same two schools; they participated in the baseline screening, but were not included in the intervention trial due to adequate Fe status. We enrolled this third group as a reference group to compare the composition of the gut microbiota between Fe-deficient and Fe-sufficient children at baseline and to follow the changes in the gut microbiota over time without the intervention.

In a previous study in Ivorian school children, a sample size of thirty children per group was adequate to detect significant differences in the major bacterial groups in the gut microbiota after $\mathrm{Fe}$ fortification of $10 \mathrm{mg} \mathrm{Fe} / \mathrm{d}^{(9)}$. Since in the present study, a much higher Fe dose ( $50 \mathrm{mg}$ Fe, $4 \mathrm{~d}$ /week) was provided via oral supplementation, we estimated that a sample size between twenty and thirty children per group would be adequate to detect the differences between Fe supplementation and placebo treatment.

Trained local fieldworkers conducted two $24 \mathrm{~h}$ dietary recalls 2 weeks apart on different days of the week. The $24 \mathrm{~h}$ dietary recalls were administered to the parents/carers of sixty children per participating school in their local language (Zulu). One recall per child was conducted for a week day and one recall was conducted for a weekend day. Dietary data were analysed with the Food Finder computer program (Medical Research Council, 2003). Anthropometric measurements were taken at baseline and endpoint, as described previously ${ }^{(3)}$. Age- and sex-specific height-for-age $z$ scores, weight-for-age $z$ scores and BMI-for-age $z$ scores were calculated using the 2007 WHO growth standards for children aged 5 to 19 years with the software WHO Anthro Plus for personal computers (version 1.0.3, WHO, 2010). Weight-for-age $z$ scores were available only for children $<11$ years of age. The present study was performed from February to November 2010 and was interrupted by holidays for 2 weeks in March and April, for 5 weeks in June and July, and for 1 week in September. Supplementation was further interrupted for 4.5 weeks by a national strike of teachers in August and September. To catch up on the unexpected loss of intervention days, supplementation was increased from 4 to $5 \mathrm{~d}$ /week for 8 weeks, which compensated for 2 weeks of supplementation. Thus, in total, supplements were provided for $105 \mathrm{~d}$ over a period of 38 weeks. The present study was conducted according to the guidelines laid down in the Declaration of Helsinki, and all procedures including human subjects were approved by the ethics committees of the North-West University in South Africa and by the Swiss Federal Institute of Technology Zürich in Switzerland. Written informed consent was obtained from parents, and verbal consent of children was witnessed and formally recorded. The present trial was registered at ClinicalTrials.gov as NCT01092377.

\section{Sample collection and blood sample analysis}

Faecal samples were collected from 09.00 to 12.00 hours at four time points throughout the study: at baseline just before the start of the intervention, and at 2 weeks, 12 weeks (midpoint) and 38 weeks (endpoint) of Fe supplementation (Fig. 1). Faecal sample collection was specifically optimised to avoid bacterial cell death by oxygen or bacterial growth due to the lack of a constant low temperature before freezing. The samples were collected immediately after defecation in zip-lock bags containing an Anaerocult A mini bag (Merck Millipore) to maintain anaerobiosis. Samples were kept at $4{ }^{\circ} \mathrm{C}$ and within $6 \mathrm{~h}$, aliquots were made in several $2 \mathrm{ml}$ Eppendorf tubes and frozen at $-80^{\circ} \mathrm{C}$ until further analysis.

Blood samples were collected at baseline and endpoint, as described previously ${ }^{(3)}$. Briefly, venous blood samples $(10 \mathrm{ml})$ were drawn into EDTA-coated and trace-element free tubes (Becton Dickinson) at baseline and endpoint. Hb concentrations were measured on site in the whole blood by the direct cyanmethaemoglobin method (Ames Mini-Pak Hb test pack and Ames Minilab; Bio-Rad Laboratories (PTY) Limited) with Drabkin's solution and a standard miniphotometer. The remaining samples were centrifuged at $500 \boldsymbol{g}$ for $15 \mathrm{~min}$ at room temperature, and plasma and serum aliquots were prepared and stored at $-20^{\circ} \mathrm{C}$ for the duration of the fieldwork ( $4 \mathrm{~d}$ ) and then at $-80^{\circ} \mathrm{C}$ until analysis. Erythrocytes were washed twice with $0.15 \mathrm{M}-\mathrm{NaCl}$ and centrifuged at $500 \boldsymbol{g}$ for 10 min to remove the buffy coat. ZnPP was determined on site on washed erythrocytes, as described previously ${ }^{(3)}$. SF, CRP and TfR concentrations were measured in serum, as described previously ${ }^{(3)}$. Fe deficiency during the intervention was defined as a SF concentration $<15 \mu \mathrm{g} / \mathrm{l}^{(32)}$ or a ZnPP concentration $>70 \mu \mathrm{mol} / \mathrm{mol}$ haem $^{(33)}$ or a TfR concentration $>8.3 \mathrm{mg} / \mathrm{l}$ (test kit reference value), and anaemia was defined as a $\mathrm{Hb}$ concentration $<115 \mathrm{~g} / \mathrm{l}^{(4)}$. For the reporting of prevalence and statistical analyses, a SF concentration $<15 \mu \mathrm{g} / 1$ was used to define Fe deficiency; for inclusion into the study, an SF concentration $<20 \mu \mathrm{g} / 1$ was used. Systemic inflammation was defined as a CRP concentration $>5 \mathrm{mg} / \mathrm{l}$.

\section{Faecal sample DNA extraction and enumeration of bacterial groups}

Faecal samples were thawed on ice and total genomic DNA was extracted using the FastDNA SPIN kit for soil (MP Biomedicals), according to the manufacturer's instructions. Bacterial groups prevalent in the gut were enumerated using specific primers for the $16 \mathrm{~S}$ ribosomal RNA gene or a functional gene (Table 1) by quantitative PCR (qPCR) analysis performed with an ABI PRISM 7500-PCR sequence detection system (Life Technologies), as described previously ${ }^{(34,35)}$. qPCR consisted of $2 \times$ SYBR Green Mastermix (Life Technologies) or $2 \times$ Kapa Sybr Fast qPCR Mastermix (Biolabo 
Table 1. Primers used to enumerate selected bacterial target groups by quantitative PCR

\begin{tabular}{|c|c|c|c|}
\hline Primers & Sequence $5^{\prime}-3^{\prime}$ & Target group & Reference \\
\hline Eub338F & 5'-ACTCCTACGGGAGGCAGCAG-3' & Total bacteria & Guo et al. ${ }^{(48)}$ \\
\hline Eub518R & 5'-ATTACCGCGGCTGCTGG-3' & & \\
\hline Bac303F & $5^{\prime}$-GAAGGTCCCCCACATTG-3' & Bacteroides spp. & Ramirez-Farias et al. ${ }^{(49)}$ \\
\hline Bfr-Femrev & 5'-CGCKACTTGGCTGGTTCAG-3' & & \\
\hline Firm934F & 5'-GGAGYATGTGGTTTAATTCGAAGCA-3' & Firmicutes & Guo et al. ${ }^{(48)}$ \\
\hline Firm1060R & $5^{\prime}$-AGCTGACGACAACCATGCAC- $3^{\prime}$ & & \\
\hline Clep866mF & 5'-TTAACACAATAAGTWATCCACCTGG-3' & Clostridium Cluster IV & Ramirez-Farias et al. ${ }^{(49)}$ \\
\hline Clep1240mR & 5'-ACCTTCCTCCGTTTTGTCAAC-3' & & \\
\hline RrecF & $5^{\prime}$-GCGGTRCGGCAAGTCTGA-3' & Roseburia spp./E. rectale & Furet et al. ${ }^{(50)}$ \\
\hline Rrec630mR & $5^{\prime}$-CCTCCGACACTCTAGTMCGAC-3' & & \\
\hline Fprau223F & 5'-GATGGCCTCGCGTCCGATTAG-3' & Faecalibacterium prausnitzii & Bartosch et al. ${ }^{(51)}$ \\
\hline Fprau420R & $5^{\prime}$-CCGAAGACCTTCTTCCTCC- $3^{\prime}$ & & \\
\hline EhalF & 5'-GCGTAGGTGGCAGTGCAA-3' & Eubacterium hallii & Ramirez-Farias et al. ${ }^{(49)}$ \\
\hline EhalR & 5'-GCACCGRAGCCTATACGG-3' & & \\
\hline dsrA_F336 & $5^{\prime}$-CTGCGAATATGCCTGCTACA-3' & $\mathrm{SRB}, d s r A$ gene & Pereyra et al. ${ }^{(52)}$ \\
\hline dsrA_R533 & 5'-TGGTCGARCTTGATGTCGTC-3' & & \\
\hline F_Lacto 05 & $5^{\prime}$-AGCAGTAGGGAATCTTCCA-3' & Lactobacillus/Pediococcus/Leuconostoc spp. & Furet et al. ${ }^{(50)}$ \\
\hline R_Lacto 04 & 5'-CGCCACTGGTGTTCYTCCATATA-3' & & \\
\hline$x f p-f w$ & 5'-ATCTTCGGACCBGAYGAGAC- $3^{\prime}$ & Bifidobacteria phosphoketolase & Cleusix et al. ${ }^{(53)}$ \\
\hline xfp-rv & 5'-CGATVACGTGVACGAAGGAC-3' & & \\
\hline Eco1457F & 5'-CATTGACGTTACCCGCAGAAGAAGC-3' & Enterobacteriaceae & Bartosch et al. ${ }^{(51)}$ \\
\hline Eco1652R & 5'-CTCTACGAGACTCAAGCTTGC-3' & & \\
\hline
\end{tabular}

SRB, sulphate-reducing bacteria; dsrA, dissimilatory sulphite-reductase subunit $A$.

Scientifics Instruments), $0 \cdot 2 \mu \mathrm{m}$ of each primer and $1 \mu \mathrm{l}$ of template genomic DNA in a total volume of $25 \mu \mathrm{l}$. Amplification consisted of an initial denaturation step at $95^{\circ} \mathrm{C}$ for 10 min (20s for Kapa Sybr Fast qPCR Mastermix) followed by forty cycles of $95^{\circ} \mathrm{C}$ for $15 \mathrm{~s}(3 \mathrm{~s})$ and $60^{\circ} \mathrm{C}$ for $1 \mathrm{~min}$ (30s). A denaturation step was added to check for amplicon specificity. The samples were analysed in duplicate and standard curves with the specific target $16 \mathrm{~S}$ ribosomal RNA gene or a functional gene were included in each run, as described previously $^{(34)}$. Data were analysed with the 7500 Fast System Sequence Detection Software (version 1.4; Life Technologies) and expressed as the log number of $16 \mathrm{~S}$ ribosomal RNA gene copies or functional gene copies/g faeces.

\section{Faecal SCFA concentration analysis}

SCFA concentrations were analysed in the faecal samples of a randomly selected subgroup of children ( $n 10$ per group) by HPLC. Briefly, 200-300 mg faeces were homogenised with $1 \mathrm{ml}$ of $0.15 \mathrm{~mm}-\mathrm{H}_{2} \mathrm{SO}_{4}$ and subsequently centrifuged at $4^{\circ} \mathrm{C}$ and $9000 \mathrm{~g}$ for $20 \mathrm{~min}$. The supernatants were diluted 1:1 with MilliQ water and filtered through a $0.45 \mu \mathrm{m}$ nylon filter (Infochroma AG) before injection. HPLC (Hitachi LaChrome; Merck) was performed using a Cation-H refill cartridge $(30 \times 4.6 \mathrm{~mm})$ connected to an Aminex ${ }^{\circledR} \mathrm{HPX}-87 \mathrm{H}$ $(300 \times 7.8 \mathrm{~mm})$ column at a flow rate of $0.4 \mathrm{ml} / \mathrm{min}$ at $40^{\circ} \mathrm{C}$ and $10 \mathrm{~mm}-\mathrm{H}_{2} \mathrm{SO}_{4}$ as the eluent solution. The samples were analysed in duplicate and data expressed as $\mu \mathrm{mol} / \mathrm{g}$ faeces.

\section{Faecal calprotectin concentration analysis}

Calprotectin concentration in the faecal samples of children was measured by immunoassay (Calprest; Eurospital S.p.A), according to the manufacturer's instructions (placebo group, $n$ 12; Fe group, $n 13$; Fe-sufficient group, $n 13$ ), at baseline, midpoint and endpoint. The samples were analysed in duplicate and data expressed as $\mathrm{mg} / \mathrm{kg}$ faeces.

\section{Statistical analyses}

Statistical analyses were performed using IBM SPSS Statistics (version 19; IBM Company). Data were checked for normal distribution and transformed, if necessary. Outliers $( \pm 3 \mathrm{SD}$ from the mean) were removed from the analysis. At baseline, all variables were compared between the groups using the one-way ANOVA with post hoc Bonferroni correction for multiple comparisons. At 2 weeks, midpoint and endpoint of the study, variables were compared between the groups (Fe group, placebo group and Fe-sufficient group) using ANCOVA with corresponding baseline values as covariates. Moreover, potential differences in qPCR data and HPLC data by intervention group only (Fe and placebo groups) over time were analysed using repeated-measures ANOVA, with the sampling time point as the within-subject variable and intervention group ( $\mathrm{Fe}$ and placebo groups) as the betweensubject factor. When significant changes over time were detected, repeated-measures ANOVA was performed for each variable between baseline and the subsequent time points within each group separately to detect deviations from baseline levels. qPCR data, TfR, ZnPP, SF, CRP and calprotectin data were log transformed for statistical analyses. $P<0.05$ was considered as significant.

\section{Results}

Iron status, inflammation and anthropometric measurements of the study subjects

Anthropometric measurements, $\mathrm{Hb}$ concentration, Fe status indices and markers of systemic (CRP) and gut (calprotectin) inflammation are shown in Table 2. At baseline, both 
Table 2. Baseline and endpoint parameters of iron status, inflammation as well as anthropometric measurements of children included in the study*

(Mean values with their standard errors; medians and maximum and minimum values)

\begin{tabular}{|c|c|c|c|c|c|c|c|}
\hline & \multicolumn{2}{|c|}{$\begin{array}{l}\text { Placebo group } \\
\quad(n 27)\end{array}$} & \multicolumn{2}{|c|}{$\begin{array}{l}\text { Fe group } \\
(n 22)\end{array}$} & \multicolumn{2}{|c|}{$\begin{array}{l}\text { Fe-sufficient } \\
\text { group ( } n 24)\end{array}$} & \multirow[b]{2}{*}{$P \dagger$} \\
\hline & Mean & $\overline{\text { SEM }}$ & Mean & $\overline{\mathrm{SEM}}$ & Mean & $\overline{\text { SEM }}$ & \\
\hline Age (years) & $9 \cdot 1$ & 0.2 & $9 \cdot 1$ & 0.3 & $8 \cdot 3$ & 0.3 & \\
\hline \multicolumn{8}{|l|}{ Sex } \\
\hline \multicolumn{8}{|l|}{ Female } \\
\hline$n$ & \multicolumn{2}{|c|}{17} & \multicolumn{2}{|c|}{11} & \multicolumn{2}{|c|}{12} & \\
\hline$\%$ & \multicolumn{2}{|c|}{61} & \multicolumn{2}{|c|}{50} & \multicolumn{2}{|c|}{50} & \\
\hline \multicolumn{8}{|l|}{$\mathrm{Hb}(\mathrm{g} / \mathrm{l})$} \\
\hline Baseline & $119 \cdot 1^{b}$ & $1 \cdot 7$ & $120 \cdot 6^{\mathrm{b}}$ & $1 \cdot 6$ & $129 \cdot 6^{\mathrm{a}}$ & $1 \cdot 1$ & \\
\hline Endpoint & $125 \cdot 8$ & 1.9 & $129 \cdot 1$ & 1.7 & & & 0.266 \\
\hline \multicolumn{8}{|c|}{ Serum transferrin receptor $(\mathrm{mg} / \mathrm{l}) \ddagger$} \\
\hline \multicolumn{8}{|c|}{ Baseline } \\
\hline Median & \multirow{2}{*}{\multicolumn{2}{|c|}{$\begin{array}{c}5 \cdot 9^{\mathrm{a}} \\
2 \cdot 8-11 \cdot 7\end{array}$}} & \multirow{2}{*}{\multicolumn{2}{|c|}{$\begin{array}{c}6 \cdot 0^{\mathrm{a}} \\
3 \cdot 3-10 \cdot 2\end{array}$}} & & & \\
\hline Minimum-maximum & & & & & 3.2 & & \\
\hline Endpoint & & & & & & & \\
\hline Median & & & & & & & $<0.001$ \\
\hline Minimum-maximum & $6 \cdot 2-$ & & 4.6 & & & & \\
\hline Zinc protoporphyrin ( $\mu \mathrm{mol} /$ & & & & & & & \\
\hline Baseline & & & & & & & \\
\hline Median & & & & & & & \\
\hline Minimum-maximum & $40 \cdot 0-$ & 1.0 & 43.0 & $7 \cdot 0$ & $31 \cdot 0$ & & \\
\hline Endpoint & & & & & & & \\
\hline Median & 77 & & & & & & 0.131 \\
\hline Minimum-maximum & $35 \cdot 5-$ & 2.5 & 40.5 & 6.0 & & & \\
\hline Serum ferritin§ $(\mu \mathrm{g} / \mathrm{l}) \ddagger$ & & & & & & & \\
\hline Baseline & & & & & & & \\
\hline Median & & & & & & & \\
\hline Minimum-maximum & $3.8-$ & & 11.5 & 4.7 & $35 \cdot 0$ & & \\
\hline Endpoint & & & & & & & \\
\hline Median & 25 & & & & & & $<0.001$ \\
\hline Minimum-maximum & $4 \cdot 4-$ & & 23.6 & 8.0 & & & \\
\hline $\operatorname{CRP}(\mathrm{mg} / \mathrm{l}) \ddagger$ & & & & & & & \\
\hline Baseline & & & & & & & \\
\hline Median & & & & & & & \\
\hline Minimum-maximum & & & & & $0-$ & & \\
\hline Endpoint & & & & & & & \\
\hline Median & & & & & & & 0.304 \\
\hline Minimum-maximum & & & & & & & \\
\hline Weight-for-age $z$ score & & & & & & & \\
\hline Baseline & 0.33 & 0.23 & -0.02 & 0.35 & & & \\
\hline Endpoint & 0.32 & 0.27 & 0.44 & 0.32 & & & 0.710 \\
\hline Height-for-age $z$ score & & & & & & & \\
\hline Baseline & -0.67 & 0.17 & -0.66 & 0.24 & & & \\
\hline Endpoint & -0.55 & 0.19 & -0.70 & 0.25 & & & 0.111 \\
\hline BMl-for-age $z$ score & & & & & & & \\
\hline Baseline & 0.61 & 0.19 & 0.35 & 0.24 & & & \\
\hline Endpoint & 0.46 & 0.18 & 0.36 & 0.24 & & & 0.293 \\
\hline Faecal calprotectin $(\mathrm{mg} / \mathrm{kg}$ & & & & & & & \\
\hline Baseline & & & & & & & \\
\hline Median & 60 & & & & & & \\
\hline Minimum-maximum & $24 \cdot 6-$ & 1.4 & $17 \cdot 3$ & $9 \cdot 1$ & $16 \cdot 8-$ & 3.8 & \\
\hline Midpoint & & & & & & & \\
\hline Median & & & & & & & 0.501 \\
\hline Minimum-maximum & $18 \cdot 4-$ & $6 \cdot 0$ & $17 \cdot 8$ & 1.3 & $16 \cdot 4-$ & & \\
\hline Endpoint & & & & & & & \\
\hline Median & $6 s$ & & & & & & 0.865 \\
\hline Minimum-maximum & $15 \cdot 6-$ & 0.5 & $17 \cdot 1$ & $6 \cdot 3$ & $19 \cdot 8-$ & 6.7 & \\
\hline
\end{tabular}

CRP, C-reactive protein.

${ }_{\mathrm{a}, \mathrm{b}}$ Mean values with unlike superscript letters were significantly different $(P<0.05$; one-way ANOVA with post hoc Bonferroni correction).

* No Fe status indices and anthropometric measurements were available for children in the Fe-sufficient group at the endpoint.

† Midpoint (only for faecal calprotectin) and endpoint variables were compared between the groups using ANCOVA with respective baseline values as covariates.

$\ddagger$ Data were log transformed for statistical analyses.

$\S$ Only those children were considered whose CRP concentrations were $<5 \mathrm{mg} / \mathrm{l}$.

|| Placebo group, $n$ 12; Fe group, $n$ 13; Fe-sufficient group, $n 13$. 
Fe-deficient groups (placebo and Fe groups) had significantly lower $\mathrm{Hb}$ and lower Fe status than the Fe-sufficient group. The prevalence of Fe deficiency based on the concentrations of TfR and SF in the Fe group was 18.2 and $9.1 \%$, respectively, and in the placebo group 18.5 and $29.6 \%$, respectively. Moreover, $25.9 \%$ of the children in the placebo group and $13.6 \%$ of the children in the Fe group were anaemic, while none of the children in the Fe-sufficient group were anaemic. There was a significant intervention effect (ANCOVA with baseline values as covariates) of Fe supplementation for lower TfR concentrations $(P<0.001)$ and higher SF concentrations $(P<0.001)$ at the endpoint in the Fe group compared with the placebo group. Fe supplementation did not affect the concentration of CRP. Faecal calprotectin concentrations did not differ between the groups at baseline, midpoint and endpoint, and were not affected by Fe supplementation.
Dietary assessment done in the study population found a mean background dietary $\mathrm{Fe}$ intake of 9.8 (SEM 0.3$) \mathrm{mg} / \mathrm{d}$. The incidence of illness was recorded during the entire trial period, and the mean days absent from school due to illness was 1.3 (SEM $0 \cdot 3) \mathrm{d}$ and due to gastrointestinal illness (diarrhoea, stomach pain and/or vomiting) was $0 \cdot 3$ (SEM $0 \cdot 1) \mathrm{d}$ in children included in the present study. Moreover, the mean days absent due to all illness and gastrointestinal illness did not differ between the Fe (all illness: 1.2 (SEM 0.4) d; gastrointestinal illness: $0 \cdot 2(\operatorname{sem} 0 \cdot 1) \mathrm{d}$ ) and placebo (all illness: $1 \cdot 3$ $(\operatorname{sem} 0 \cdot 4) \mathrm{d}$; gastrointestinal illness: $0 \cdot 3(\operatorname{sem} 0 \cdot 2) \mathrm{d})$ groups.

\section{Concentrations of dominant bacterial groups}

Total 16S ribosomal RNA gene copies were stable over the entire trial period, and no differences were observed between the treatment groups across the different time points (Fig. 2).

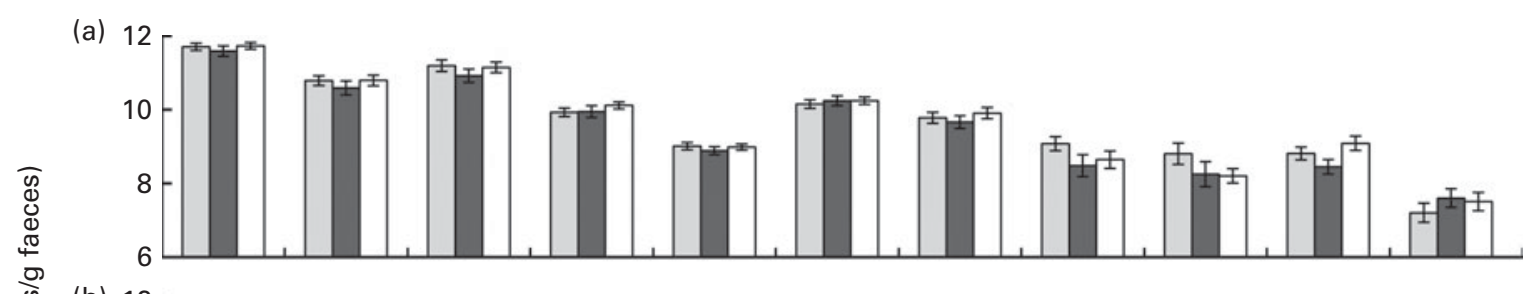

(b) 12

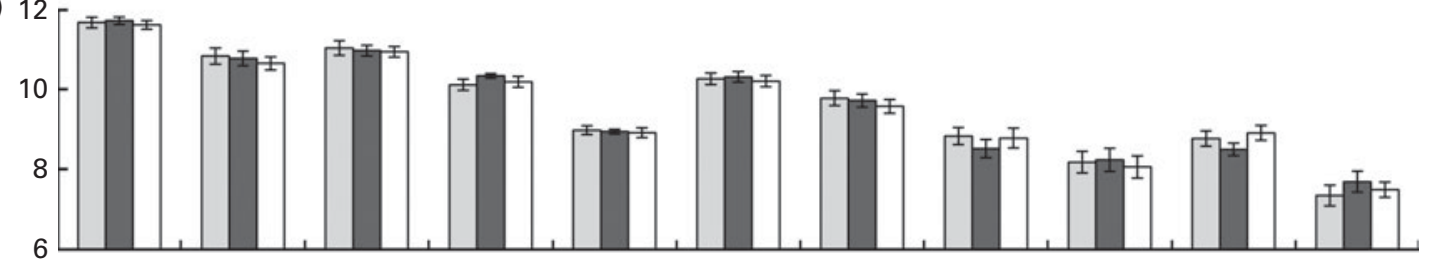

(c)

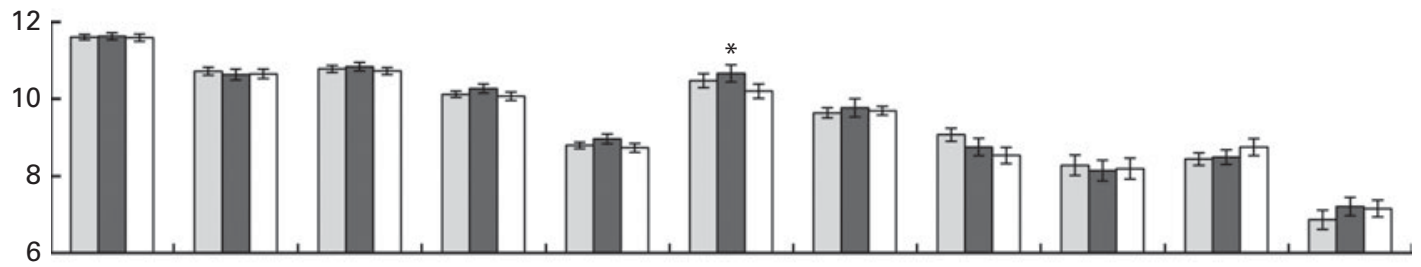

(d) 12

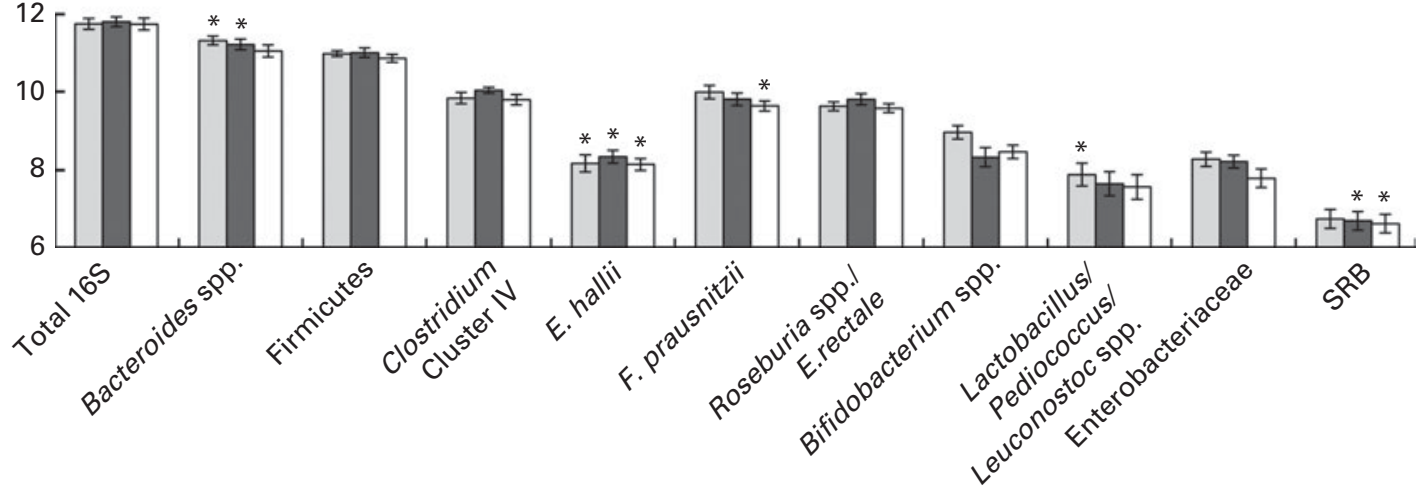

Fig. 2. Log number of $16 \mathrm{~S}$ ribosomal RNA gene copies or functional gene copies/g faeces of selected bacterial target groups in the gut microbiota of children in the placebo group ( $\square$ ), iron group ( $\square$ ) and iron-sufficient group ( $\square$ ) at (a) baseline, (b) 2 weeks, (c) midpoint and (d) endpoint. No significant treatment $\times$ time interaction was detected using repeated-measures ANOVA, with the sampling time point as the within-subject variable and intervention group (iron and placebo groups) as the between-subject factor. Values are means, with their standard errors represented by vertical bars. ${ }^{*}$ Mean value was significantly different from that of baseline concentrations of the same bacterial target group within a treatment group ( $P<0.05$; repeated-measures ANOVA). SRB, sulphate-reducing bacteria. 
At baseline, measured gut bacterial populations did not differ between the children who were Fe deficient (placebo and $\mathrm{Fe}$ groups) and those with an adequate $\mathrm{Fe}$ status (Fe-sufficient group). There was a trend observed towards lower concentrations of Enterobacteriaceae in the faeces of children in the Fe group compared with the Fe-sufficient group $(P=0.064)$.

With baseline concentrations as covariates, there were no significant differences observed between the groups in terms of concentrations of any of the measured bacterial target groups at 2 weeks, midpoint or endpoint of the study. Moreover, no significant effects of Fe treatment or timex treatment interaction were observed when analysing each bacterial target group over time and including only the placebo and Fe groups in the analysis. However, there were significant effects for time within the groups, when comparing bacterial concentrations at 2 weeks, midpoint and endpoint with baseline. As shown in Fig. 2, Bacteroides spp. significantly increased in the placebo $(P=0.004)$ and $\mathrm{Fe}(P=0.004)$ groups from baseline to the endpoint, while Eubacterium hallii concentrations significantly decreased in the placebo $(P<0.001), \quad \mathrm{Fe} \quad(P=0.024)$ and Fe-sufficient $(P<0.001)$ groups, and sulphate-reducing bacteria concentrations significantly decreased only in the Fe-sufficient $(P=0.03)$ and $\mathrm{Fe}$ $(P=0.008)$ groups from baseline to the endpoint. Concentrations of Lactobacillus/Leuconostoc/Pediococcus spp. significantly decreased from baseline to the endpoint $(P=0.027)$ in the placebo group, and Faecalibacterium prausnitzii concentrations decreased from baseline to the endpoint $(P=0.004)$ in the Fe-sufficient group. Furthermore, concentrations of $F$. prausnitzii increased from baseline to the midpoint $(P=0.045)$ in the Fe group, which was not observed in the placebo group or the Fe-sufficient group (Fig. 3).

\section{Faecal SCFA concentrations}

Baseline faecal acetate, propionate and butyrate concentrations did not differ between the placebo, Fe and the Fe-sufficient groups (Fig. 4(a)-(c), respectively). Also, the baseline ratios of acetate:propionate:butyrate did not differ between the placebo (59:28:13), Fe (58:27:15) and Fe-sufficient (56:30:14) groups.

With baseline values as covariates, there were no significant differences observed in faecal acetate, propionate and butyrate concentrations between the three groups at 2 weeks, midpoint or endpoint of the study. Furthermore, no significant effects for Fe treatment and timeXtreatment interaction were found for SCFA production by the gut microbiota when including only the Fe and placebo groups in the analysis. However, there were significant effects for time within the Fe and placebo groups (but not within the Fe-sufficient group) when comparing the concentrations of metabolites during the intervention with those at baseline. Acetate concentrations significantly increased from baseline to 2 weeks $(P=0.026)$ in the Fe group (Fig. 4(a)). In the placebo group, acetate concentrations significantly increased from baseline to the endpoint $(P=0.009)$. In the Fe and placebo groups, butyrate concentrations significantly increased from baseline

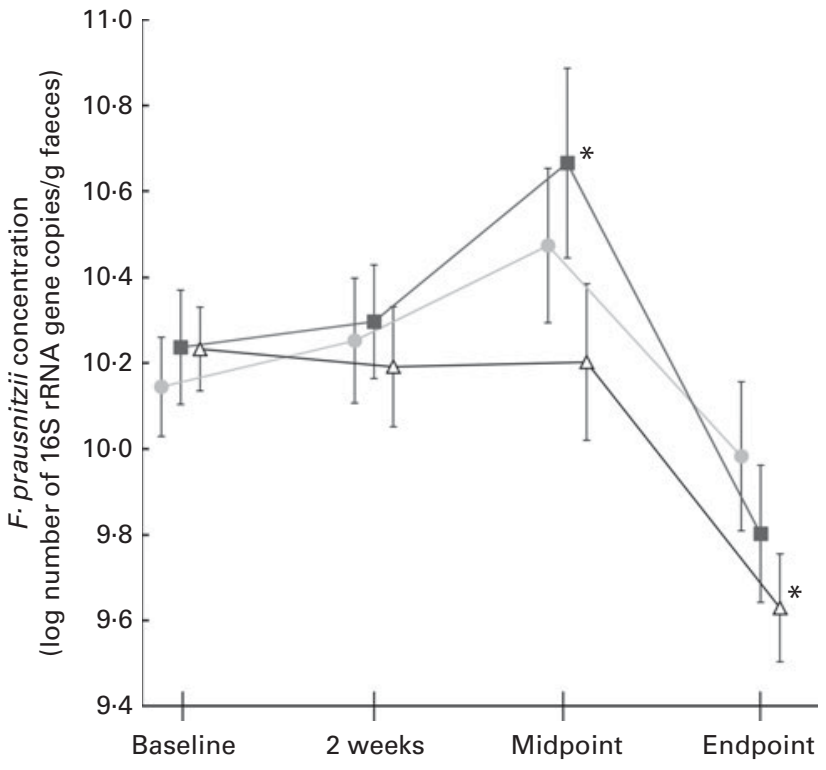

Fig. 3. Log number of $16 \mathrm{~S}$ ribosomal RNA (rRNA) gene copies/g faeces of Faecalibacterium prausnitzii in the faecal samples of children in the placebo group ( $n$ 27, -o), iron group ( $n$ 22, -7 ) and iron-sufficient group $(n 24,-\checkmark)$ measured over time by quantitative PCR. No significant treatment $\times$ time interaction was detected using repeated-measures ANOVA, with the sampling time point as the within-subject variable and intervention group (iron and placebo groups) as the between-subject factor. Values are means, with their standard errors represented by vertical bars. * Mean value was significantly different from that of baseline concentrations within a treatment group ( $P<0.05$; repeated-measures ANOVA).

to 2 weeks $(P=0.001$ and $P=0.002$, respectively) and to the endpoint $(P=0.034$ and $P=0.040$, respectively; Fig. $4(\mathrm{c}))$. At midpoint, butyrate concentrations remained significantly higher than those at baseline in the Fe group $(P=0.040)$. There were no significant effects of time on propionate concentrations within the groups (Fig. 4(b)).

\section{Discussion}

The present study was the first randomised, placebocontrolled $\mathrm{Fe}$ intervention trial to investigate the impact of oral Fe supplementation on gut microbiota and gut inflammation in African children. The major finding of the present study is that high-dose $\mathrm{Fe}$ supplementation $(50 \mathrm{mg} \mathrm{Fe}$, $4 \mathrm{~d}$ /week) over a period of 38 weeks did not significantly modify the concentrations of dominant bacterial groups in the gut or faecal SCFA, and did not increase gut inflammation. A second important finding is that the dominant bacterial groups prevalent in the gut and faecal SCFA concentrations of Fe-sufficient children were not different from those of Fe-deficient children.

We found no effect of Fe supplementation on the abundance of dominant bacterial groups in the gut compared with the placebo treatment. These findings are in sharp contrast to the previous study in similarly aged children living in rural Côte d'Ivoire, where daily provision of Fe-fortified biscuits (10 mg Fe as electrolytic Fe) to school children for 6 months significantly increased Enterobacteriaceae and decreased lactobacilli concentrations ${ }^{(9)}$. This difference may 

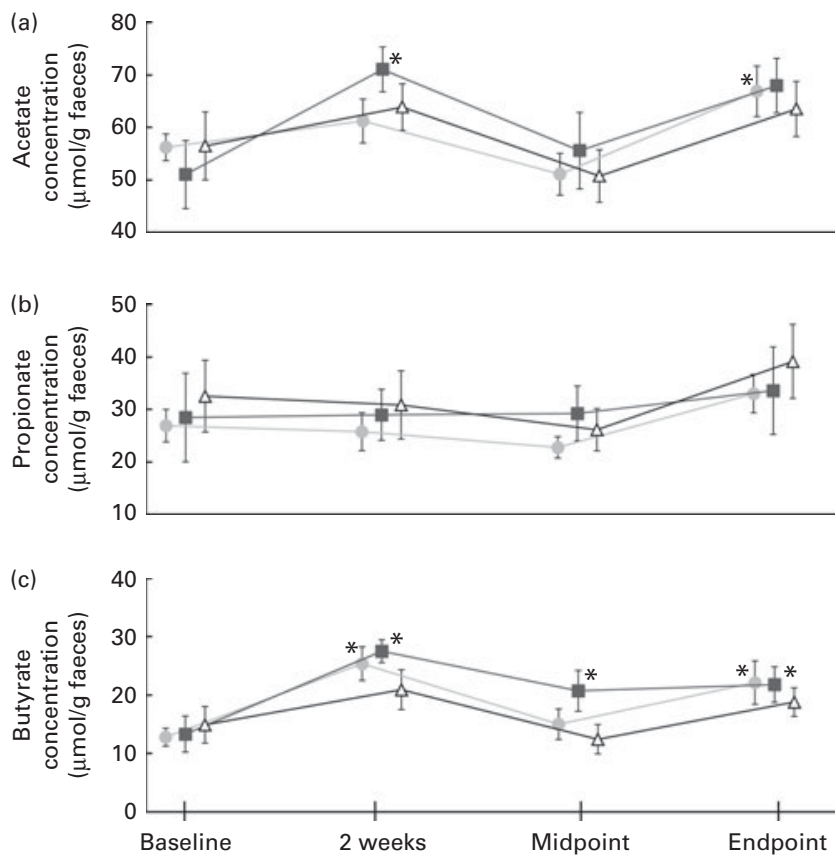

Fig. 4. (a) Acetate, (b) propionate and (c) butyrate concentrations in the faecal samples of children in the placebo group (-O), iron group ( $\square$ ) and iron-sufficient group $\left(-\triangle_{-}\right)$at baseline, 2 weeks, midpoint and endpoint of the study. No significant treatment $\times$ time interaction was detected using repeated-measures ANOVA, with the sampling time point as the within-subject variable and intervention group (iron and placebo groups) as the between-subject factor. Values are means $(n 9-10$ children per group and time point), with their standard errors represented by vertical bars. * Mean value was significantly different from that of baseline concentrations of the same metabolite within a treatment group $(P<0.05$; repeated-measures ANOVA).

be at least partially explained by the fact that Ivorian children lived in a remote rural area with poorer-quality water and food sanitation, a more monotonous low-quality diet and a higher infectious disease burden (including enteropathogens and malaria). Colonic Fe can promote the growth and virulence of certain pathogens ${ }^{(36-38)}$. During enteropathogen infection and the resulting inflammation, the composition of the gut microbiota is shifted towards facultative anaerobes such as enterobacteria ${ }^{(29,39-42)}$, and high colonic $\mathrm{Fe}$ concentrations may contribute to these effects by aggravating inflammation $^{(18,43)}$. The children in the present study were mainly from households with access to relatively clean tap water and lived in a malaria-free environment. In addition, the schools in this area of South Africa participated in the National School Nutrition Program, where children were offered a daily school meal that contributes to nutritional diversity. Nevertheless, studies in infants have shown an impact of additional dietary Fe supplementation on the gut microbiota even in industrialised countries with a low enteropathogen burden ${ }^{(26,44)}$. However, the gut microbiota in infants is in the process of developing into a fully diversified bacterial ecosystem. This still fragile bacterial consortium may be more vulnerable to alterations in luminal Fe status than a fully diversified and stable gut microbiota such as that found in the 6- to 11-year-old children of the present study.
We also found that the abundance of dominant bacterial groups and faecal SCFA concentrations did not differ between Fe-deficient ( $\mathrm{Fe}$ and placebo groups) and Fe-sufficient children. This is in contrast with several animal studies that associated Fe deficiency with changes in the composition of the gut microbiota ${ }^{(17,18,24)}$. Animal and in vitro studies have further shown that Fe deficiency modifies the metabolic activity of the gut microbiota, resulting in a decrease in butyrate production ${ }^{(21,34)}$. These differences from our findings may be due to the differences in the severity of Fe deficiency among the studies. Most of the children included in the present study were only mildly Fe deficient. South Africa fortifies wheat flour and maize meal with Fe, and the mean daily Fe intake in the present study population was found to be $9 \cdot 8$ ( $\operatorname{sem} 0 \cdot 3) \mathrm{mg}^{(3,45)}$. Thus, colonic luminal Fe concentrations in children of the present study were unlikely to have been very low. In previous in vitro studies, we have shown that a dietary Fe concentration in this range is sufficient to maintain a stable gut microbiota $^{(34)}$.

During the study, there was an overall decrease observed in the concentrations of faecal lactobacilli, E. hallii, F. prausnitzii and sulphate-reducing bacteria, while concentrations of Bacteroides spp. slightly increased from baseline to the endpoint in all the treatment groups. Since these changes were independent of $\mathrm{Fe}$ supplementation and also observed in the Fe-sufficient group, we assume that either seasonal changes or other alterations in dietary habits during the intervention (including a long school vacation between the midpoint and the endpoint, with no school lunch provided) may be responsible for these time effects.

SCFA acetate, propionate and butyrate are the major metabolites of the gut microbiota. In the present study, no differences in SCFA were observed between Fe-sufficient and Fe-deficient children at baseline, and Fe supplementation did not affect faecal SCFA concentrations. This is in contrast to previous studies in rats, where Fe supplementation increased the metabolic activity of the microbiota, particularly butyrate ${ }^{(21,22)}$. However, generally, only about $5 \%$ of the SCFA produced by the gut microbiota are excreted in the faeces; the remainder are readily absorbed by the host, which can lead to large variations in faecal SCFA concentrations ${ }^{(46)}$. Nevertheless, faecal acetate or butyrate concentrations were modified in the Fe and placebo groups over time, which may be explained by seasonal alterations in diet or other factors, as discussed above, for the composition of the gut microbiota.

Because high luminal Fe concentrations can promote inflammation ${ }^{(18,43)}$, in the present study, we assessed systemic inflammation by serum CRP and local colonic inflammation by faecal calprotectin, a peptide secreted by neutrophils infiltrating the gut mucosa. Serum CRP and faecal calprotectin concentrations did not differ between Fe-sufficient and $\mathrm{Fe}$ deficient children at baseline. Furthermore, in this setting, high-dose $\mathrm{Fe}$ supplementation in Fe-deficient children did not measurably increase systemic or gut inflammation. Thus, our findings differ from the study conducted in rural Côte d'Ivoire, where provision of Fe-fortified biscuits increased faecal calprotectin concentrations compared with a control group $^{(9)}$. However, in that study, Fe fortification increased 
enterobacteria numbers (including many potential enteropathogens causing gut inflammation), which correlated with faecal calprotectin. In the present study, the overall incidence of diarrhoea during the intervention was very low with an average of only $0 \cdot 1(\operatorname{sem} 0 \cdot 1) \mathrm{d}$ absent due to gastrointestinal illness, and no differences between $\mathrm{Fe}$ - and placebosupplemented children were observed. However, clinical data should be interpreted with caution, as they were based on self-reporting and the sample size of the present study was small. Calprotectin concentrations in our children were generally higher than those previously reported in African children at this age ${ }^{(9,47)}$

In summary, in South African school-aged children from a malaria-free rural area with a low gastrointestinal disease burden, we found no significant differences in the abundance of dominant bacterial groups or faecal SCFA concentrations in mildly Fe-deficient and non-Fe-deficient children. This suggests that dietary and luminal Fe levels in both groups were sufficient to maintain the gut microbiota. Furthermore, high-dose Fe supplementation had no measurable impact on the abundance of dominant bacterial groups in the gut, faecal SCFA concentration or gut inflammation. Therefore, it appears that Fe supplementation poses a low risk for negative modulation of the tested bacterial groups and/or adverse intestinal effects at this age and in this setting. Hence, our initial hypothesis that high-dose Fe supplementation would modify the tested bacterial groups and metabolites under all conditions could not be confirmed. The effects of $\mathrm{Fe}$ supplementation on the gut microbiota most probably also depend on environmental factors (e.g. presence of enteropathogens) and gut inflammatory preset of the host. Future research should therefore investigate the potential effects of $\mathrm{Fe}$ supplementation on the gut microbiota in other age groups, in populations where Fe deficiency is more severe, and in settings where poor-quality water and food supplies increase exposure to potential enteropathogens.

\section{Acknowledgements}

The authors thank the fieldworkers, teachers and principals of the schools for their support of the study; the children and parents for their participation in the trial; the Medical Research Council and North-West University colleagues, especially Linda Malan and Ellenor Rossouw, and students, especially Jani Greeff, for their assistance during field and laboratory work; Nicola Mosca and Van Pham Thanh for assistance with the composition of the gut microbiota and faecal SCFA concentration analyses.

The present study was supported by the Swiss National Science Foundation (project no.: 310030_127272, Bern, Switzerland); Eunice Kennedy Shriver National Institute of Child Health and Human Development (award no.: U01HD0 64921); and Unilever Research and Development, Vlaardingen, The Netherlands. Paul Lohmann GmbH (Lomapharm, Emmertal, Germany) provided the Fe supplements. Travel support was provided by the European-South African Partnership in Nutrition Research. None of the funders had a role in the design, analysis or writing of this article.
The authors' contributions are as follows: A. D., J. B., C. C., C. M. S., M. B. Z. and C. L. designed the research; A. D., J. B. and N. R. conducted the research; A. D., J. B. and N. R. analysed the data; A. D., J. B., C. C., M. B. Z. and C. L. wrote the paper; C. L. had primary responsibility for the final content. All authors read and approved the final manuscript.

None of the authors had a conflict of interest to declare.

\section{References}

1. Zimmermann MB \& Hurrell RF (2007) Nutritional iron deficiency. Lancet 370, 511-520.

2. Beard J (2003) Iron deficiency alters brain development and functioning. J Nutr 133, 1468S-1472S.

3. Baumgartner J, Smuts CM, Malan L, et al. (2012) Effects of iron and $n-3$ fatty acid supplementation, alone and in combination, on cognition in school children: a randomized, double-blind, placebo-controlled intervention in South Africa. Am J Clin Nutr 96, 1327-1338.

4. WHO (2002) Iron Deficiency Anemia: Assessment, Prevention, and Control. A Guide for Programme Managers. Geneva: World Health Organization.

5. Stoltzfus RJ \& Dreyfuss ML (1998) Guidelines for the Use of Iron Supplements to Prevent and Treat Iron Deficiency Anemia. Washington, DC: ILSI Press.

6. Gera T \& Sachdev HP (2002) Effect of iron supplementation on incidence of infectious illness in children: systematic review. BMJ 325, 1142.

7. Iannotti LL, Tielsch JM, Black MM, et al. (2006) Iron supplementation in early childhood: health benefits and risks. Am J Clin Nutr 84, 1261-1276.

8. Hurrell RF (2011) Safety and efficacy of iron supplements in malaria-endemic areas. Ann Nutr Metab 59, 64-66.

9. Zimmermann MB, Chassard C, Rohner F, et al. (2010) The effects of iron fortification on the gut microbiota in African children: a randomized controlled trial in Cote d'Ivoire. $\mathrm{Am}$ J Clin Nutr 92, 1406-1415.

10. Sekirov I, Russell SL, Antunes LC, et al. (2010) Gut microbiota in health and disease. Physiol Rev 90, 859-904.

11. Flint HJ, Scott KP, Louis P, et al. (2012) The role of the gut microbiota in nutrition and health. Nat Rev Gastroenterol Hepatol 9, 577-589.

12. Gordon JI, Dewey KG, Mills DA, et al. (2012) The human gut microbiota and undernutrition. Sci Transl Med 4, 137ps112.

13. Yatsunenko T, Rey FE, Manary MJ, et al. (2012) Human gut microbiome viewed across age and geography. Nature 486, 222-227.

14. Smith MI, Yatsunenko T, Manary MJ, et al. (2013) Gut microbiomes of Malawian twin pairs discordant for kwashiorkor. Science 339, 548-554.

15. Monira S, Nakamura S, Gotoh K, et al. (2011) Gut microbiota of healthy and malnourished children in Bangladesh. Front Microbiol 2, 228.

16. Gupta SS, Mohammed MH, Ghosh TS, et al. (2011) Metagenome of the gut of a malnourished child. Gut Pathog 3, 7.

17. Tompkins GR, O'Dell NL, Bryson IT, et al. (2001) The effects of dietary ferric iron and iron deprivation on the bacterial composition of the mouse intestine. Curr Microbiol 43 , $38-42$.

18. Werner T, Wagner SJ, Martinez I, et al. (2011) Depletion of luminal iron alters the gut microbiota and prevents Crohn's disease-like ileitis. Gut 60, 325-333.

19. Lee SH, Shinde P, Choi J, et al. (2008) Effects of dietary iron levels on growth performance, hematological status, liver mineral concentration, fecal microflora, and diarrhea 
incidence in weanling pigs. Biol Trace Elem Res 126, Suppl. 1, S57-S68.

20. Benoni G, Cuzzolin L, Zambreri D, et al. (1993) Gastrointestinal effects of single and repeated doses of ferrous sulphate in rats. Pharmacol Res 27, 73-80.

21. Dostal A, Chassard C, Hilty FM, et al. (2012) Iron depletion and repletion with ferrous sulfate or electrolytic iron modifies the composition and metabolic activity of the gut microbiota in rats. $J$ Nutr 142, 271-277.

22. Dostal A, Lacroix C, Pham VT, et al. (2014) Iron supplementation promotes gut microbiota metabolic activity but not colitis markers in human gut microbiota-associated rats. $\mathrm{Br}$ J Nutr 21, 1-11.

23. Andrews SC, Robinson AK \& Rodriguez-Quinones F (2003) Bacterial iron homeostasis. FEMS Microbiol Rev 27, 215-237.

24. Balamurugan R, Mary RR, Chittaranjan S, et al. (2010) Low levels of faecal lactobacilli in women with iron-deficiency anaemia in south India. Br J Nutr 104, 931-934.

25. Mevissen-Verhage EA, Marcelis JH, Harmsen-van Amerongen WC, et al. (1985) Effect of iron on neonatal gut flora during the first week of life. Eur J Clin Microbiol 4, 14-18.

26. Krebs NF, Sherlock LG, Westcott J, et al. (2013) Effects of different complementary feeding regimens on iron status and enteric microbiota in breastfed infants. $J$ Pediatr $\mathbf{1 6 3}$, 416-423.

27. Weinberg ED (2009) Iron availability and infection. Biochim Biophys Acta 1790, 600-605.

28. Doherty CP (2007) Host-pathogen interactions: the role of iron. J Nutr 137, 1341-1344.

29. Stecher B \& Hardt WD (2008) The role of microbiota in infectious disease. Trends Microbiol 16, 107-114.

30. Soofi S, Cousens S, Iqbal SP, et al. (2013) Effect of provision of daily zinc and iron with several micronutrients on growth and morbidity among young children in Pakistan: a clusterrandomised trial. Lancet 382, 29-40.

31. Cunnington AJ, de Souza JB, Walther M, et al. (2012) Malaria impairs resistance to Salmonella through heme- and heme oxygenase-dependent dysfunctional granulocyte mobilization. Nat Med 18, 120-127.

32. Zimmermann MB (2008) Methods to assess iron and iodine status. Br J Nutr 99, Suppl. 3, S2-S9.

33. Metzgeroth G, Adelberger V, Dorn-Beineke A, et al. (2005) Soluble transferrin receptor and zinc protoporphyrin - competitors or efficient partners? Eur J Haematol 75, 309-317.

34. Dostal A, Fehlbaum S, Chassard C, et al. (2013) Low iron availability in continuous in vitro colonic fermentations induces strong dysbiosis of the child gut microbial consortium and a decrease in main metabolites. FEMS Microbiol Ecol 83, 161-175

35. Zihler A, Gagnon M, Chassard C, et al. (2010) Unexpected consequences of administering bacteriocinogenic probiotic strains for Salmonella populations, revealed by an in vitro colonic model of the child gut. Microbiology 156, $3342-3353$.

36. Bjarnason J, Southward CM \& Surette MG (2003) Genomic profiling of iron-responsive genes in Salmonella enterica serovar Typhimurium by high-throughput screening of a random promoter library. J Bacteriol 185, 4973-4982.

37. Layton AN, Hudson DL, Thompson A, et al. (2010) Salicylidene acylhydrazide-mediated inhibition of type III secretion system-1 in Salmonella enterica serovar Typhimurium is associated with iron restriction and can be reversed by free iron. FEMS Microbiol Lett 302, 114-122.
38. Janakiraman A \& Slauch JM (2000) The putative iron transport system SitABCD encoded on SPI1 is required for full virulence of Salmonella typhimurium. Mol Microbiol 35, 1146-1155.

39. Raffatellu M, George MD, Akiyama Y, et al. (2009) Lipocalin2 resistance confers an advantage to Salmonella enterica serotype Typhimurium for growth and survival in the inflamed intestine. Cell Host Microbe 5, 476-486.

40. Winter SE, Thiennimitr P, Winter MG, et al. (2010) Gut inflammation provides a respiratory electron acceptor for Salmonella. Nature 467, 426-429.

41. Winter SE, Lopez CA \& Baumler AJ (2013) The dynamics of gut-associated microbial communities during inflammation. EMBO Rep 14, 319-327.

42. Winter SE, Winter MG, Xavier MN, et al. (2013) Host-derived nitrate boosts growth of $E$. coli in the inflamed gut. Science 339, 708-711.

43. Carrier J, Aghdassi E, Platt I, et al. (2001) Effect of oral iron supplementation on oxidative stress and colonic inflammation in rats with induced colitis. Aliment Pharmacol Ther 15, 1989-1999.

44. Mevissen-Verhage EA, Marcelis JH, Harmsen-Van Amerongen WC, et al. (1985) Effect of iron on neonatal gut flora during the first three months of life. Eur J Clin Microbiol $\mathbf{4}$, $273-278$.

45. South Africa Department of Health (2003) Regulations relating to the fortification of certain foodstuffs. http:// www.doh.gov.za/docs/regulations/2003/ffortification.html

46. Cummings JH \& Macfarlane GT (1991) The control and consequences of bacterial fermentation in the human colon. J Appl Bacteriol 70, 443-459.

47. Hestvik E, Tumwine JK, Tylleskar T, et al. (2011) Faecal calprotectin concentrations in apparently healthy children aged 0-12 years in urban Kampala, Uganda: a community-based survey. BMC Pediatr 11, 9.

48. Guo X, Xia X, Tang R, et al. (2008) Development of a realtime PCR method for Firmicutes and Bacteroidetes in faeces and its application to quantify intestinal population of obese and lean pigs. Lett Appl Microbiol 47, 367-373.

49. Ramirez-Farias C, Slezak K, Fuller Z, et al. (2009) Effect of inulin on the human gut microbiota: stimulation of Bifidobacterium adolescentis and Faecalibacterium prausnitzii. Br J Nutr 101, 541-550.

50. Furet JP, Firmesse O, Gourmelon M, et al. (2009) Comparative assessment of human and farm animal faecal microbiota using real-time quantitative PCR. FEMS Microbiol Ecol 68 , $351-362$.

51. Bartosch S, Woodmansey EJ, Paterson JC, et al. (2005) Microbiological effects of consuming a synbiotic containing Bifidobacterium bifidum, Bifidobacterium lactis, and oligofructose in elderly persons, determined by real-time polymerase chain reaction and counting of viable bacteria. Clin Infect Dis 40, 28-37.

52. Pereyra LP, Hiibel SR, Prieto Riquelme MV, et al. (2010) Detection and quantification of functional genes of cellulose-degrading, fermentative, and sulfate-reducing bacteria and methanogenic archaea. Appl Environ Microbiol 76, 2192-2202.

53. Cleusix V, Lacroix C, Dasen G, et al. (2010) Comparative study of a new quantitative real-time PCR targeting the xylulose-5-phosphate/fructose-6-phosphate phosphoketolase bifidobacterial gene (xfp) in faecal samples with two fluorescence in situ hybridization methods. J Appl Microbiol 108, 181-193. 